\title{
GEOGRAPHIC ORIGINS OF INVASIVE ALIEN SPECIES IN “IRON GATES” NATURAL PARK (BANAT, ROMANIA)
}

\author{
Irina GOIA *, Cristiana-Maria CIOCANEA ** \\ and Athanasios-Alexandru GAVRILIDIS **
}

\begin{abstract}
* “Babeş-Bolyai” University, Faculty of Biology and Geology, Department of Ecology and Taxonomy, Gheorghe Bilașcu Street 44, Cluj-Napoca, Cluj County, Romania, RO-400015, irina.goia@ubbcluj.ro ** University of Bucharest, Centre for Environmental Research and Impact Studies, Nicolae Bălcescu Boulevard 1, Sector 1, Bucharest, Romania, RO-010041, cristianamaria.ciocanea@g.unibuc.ro, athanasiosalexandru.gavrilidis@g.unibuc.ro
\end{abstract}

DOI: 10.1515/trser-2015-0036

KEYWORDS: geographic origin, biogeographic dispersal, invasive alien species, "Iron Gates” Natural Park, Romania.

\section{ABSTRACT}

The paper presents an inventory and distribution of invasive alien species, in "Iron Gates” Natural Park, especially to highlight their origins, the most aggressive alien species, and their impact on conservation status of habitats, and indirectly their economic and sociological impact on the human communities.

This study may have an important role in improving the efficiency of conservation measures, offering valuable information to authorities involved in protected areas administration.

RESUMEN: Orígen geográfico de las especies invasoras en el Parque Natural "Puertas de Hierro" (Banat, Rumania).

En este trabajo se presenta un inventario de las especies invasoras y su distribución espacial en el Parque Nacional Puertas de Hierro, con el fin de establecer su origen geográfico, de identificar a las más agresivas así como también de establecer qué impacto tienen tanto en el estado de conservación de los hábitats del parque como en los aspectos socioeconómicos de las comunidades humanas que habitan el lugar.

Este estudio es potencialmente importante para mejorar la eficiencia de las medidas de manejo y conservación, y presenta información de utilidad a las autoridades involucradas en la administración de áreas protegidas.

REZUMAT: Apartenența areal geografică a speciilor invazive din Parcul Natural Porțile de Fier, România.

Lucrarea prezintă un inventar al speciilor invazive și distribuția acestora în Parcul Natural Porțile de Fier, în special pentru a sublinia apartenența areal geografică, cele mai agresive specii invazive și impactul acestora asupra stării de conservare a habitatelor, precum și impactul lor economic și sociologic asupra comunităților umane.

Acest studiu reprezintă un pas necesar în îmbunătățirea măsurilor de conservare și oferă informații valoroase autorităților implicate în administrarea ariilor protejate. 


\section{INTRODUCTION}

Invasive alien species (IAS) pose a threat to global ecosystems, and they are a major environmental threat of the 21st Century (Mack et al., 2000). Impacts include loss of native species and habitat, economic suppression, reduced food and water security, and direct threats to human health.

For many centuries, humans have intentionally or accidentally moved organisms around the planet (Elton, 1958) which resulted in many changes within world ecosystems. This was possible due to self-sustaining species subsiding outside their native ranges (Richardson, 2011), but many introduced species became invasive and have negative effects on native species and habitat structure (Gaertner et al., 2009).

Invasive species are commonly non-native species that disseminate out of their natural range generally as a result of human activities. Are incapable to live in a foreign environment without anthropic intervention and eventually die off, but due to acclimatization, humans can cause significant changes on species' ranges (Evans et al., 2008). Some species adapt to new surroundings and establish in the local ecosystems, where they can cause ecological and economic damage.

The EC estimated in 2014 that in Europe there were already 12,000 alien species, of which $10-15 \%$ are invasive. They appear in all major taxonomic groups, ranging from mammals, amphibians, reptiles, fish, invertebrates and plants to fungi, bacteria and other micro-organisms. They are found in all habitats, both on land and in water seas. (EEA, 2014)

Terrestrial plants are the most common alien species, representing over half of all species presented in Europe (over 6,500 species), followed by terrestrial invertebrates (over 2,700 species). There are close to 1,000 alien aquatic species, on the other hand, terrestrial vertebrates are the less numerous alien species present in EU. (Daisie, 2009)

Invasive alien species can cause local extinction of indigenous species, through competition for limited resources (food and habitat), interbreeding or the spread of exotic diseases. The lack of studies and sustainable actions to conserve natural protected areas, can lead to invasive alien species impacting entire ecosystems, thus altering their structure and function, including their ability to proved valuable ecosystem services, such as water regulation, flood control or even pollination. (Kumschick et al., 2012)

Invasive alien species can also have a significant economic impact, by reducing yields from agriculture, forestry and fisheries, can damage infrastructure (decrease water availability, block waterways, clog pipes). They can also destroy bodies of water bodies and change landscapes by causing loss of protected areas or cultural heritage values. Other than this, invasive alien species can be a major issue for human health, triggering skin problems or allergies and acting as a vector for other dangerous pathogens and diseases. (Mack et al., 2000)

The cost management of taking measures to combat invasive alien species is a continuous growth process. Once an invasive alien species is out of their contained environment by natural or anthropogenic reasons, human and financial resources are needed in order to repair the damage they cause, and take measures to eradicate them or stop them from spreading further. It's obvious that sooner the measures are taken the better. The Regulation on Invasive Alien species established by EU in 2014 includes three types of measure for combating IAS: prevention, early warning and rapid response and management of already established invasive alien species. (EEA, 2014) 
It is also significant to mention that not all alien species are problematic or invasive; some of them generate economic benefits, this being one of the reasons why they were introduced in different areas (Daleo et al., 2009; Donaldson et al., 2014).

The study was focused on "Iron Gates" Natural Park, located in the South-Western part of Romania, on the territory of Caraș-Severin and Mehedinți counties part of Continental biogeographical region. Considered the third largest protected area in Romania $\left(1,156 \mathrm{~km}^{2}\right)$ it is recognized for its great diversity of ecosystems, wide variety of species and emblematic landscapes.

"Iron Gates" Natural Park area was the subject of numerous complex research studies increasing before and after construction of "Iron Gates" Navigation System - Hydro Power Plants (1965-1971) (Boiangiu, 2002; Călinescu and Iana, 1964; Boșcaiu et al., 1971; etc.). Due to its importance represented by high diversity of species and ecosystems, the latest research studies focused on the conservation of natural habitats and species (Matacă, 2005; Pătroescu et al., 2008; etc.).

According to Law 5/2000, to approve the National Territory Improvement Plan Section III - protected area, M. O. 552/2003 of M.A.P.A.M., HG 2151/2004 and OUG 57/2007 in the "Iron Gates" Natural Park are 18 natural protected areas (national reserves and nature monuments). Also according to HG 1284/2007 on the territory of "Iron Gates" Natural Park, which established two Special Protection Areas (SPAs) as integrated parts of European Ecological network NATURA 2000 in Romania: ROSPA0026 - Danube water course Baziaș"Iron Gates" and ROSPA0080 Almăj-Locvei Mountains. In 2007 according to the Ministry of Environment and Sustainable Development Ordinance no. 1964 was established ROSCI0206 "Iron Gates" as a Site of Community Interest (SCI) as an integrated part of European Ecological Network NATURA 2000.

Botanical surveys carried out in "Iron Gates" Natural Park lead to a floristic inventory of 1,875 vascular plant taxa, including 1,748 species, 120 subspecies and six varieties, distributed in 570 genera and 131 families (Matacă, 2005). This floristic inventory represents 49.97\% of all floral species known in Romania (Matacă, 2005). The diversity of the vegetal associations is high, being identified 171 vegetal associations, from which 26 are endemic for Romania and 21 have community value. The number of endemic elements completes the large diversity of fitogeographic elements. Also 27 habitat types from the 29 total habitats identified are habitats of community interest, and are listed in the 1st Annex of the Habitat Directive (92/43/EEC). Moreover, five of these habitats are considered priority habitats and designated that the area needed special conservation (PM of PNPF, 2013).

Regarding the fauna of "Iron Gates" Natural Park the existent studies, reveal the presence of 5,205 taxa, of which 4,873 are invertebrates and 332 vertebrates. Among vertebrates, class Aves has the most representatives (205 taxa), followed by Pisces with 63 representatives, and class Amphibia with the lowest representativeness - only 12 taxa (PNPF Management Plan, 2013). Most of the reptiles, amphibians and birds species from the "Iron Gate" Natural Park are protected at an international and national level. The "Iron Gate" area represents also a passage corridor for birds with world importance. 
Being an EU member, the alignment of EU legislation and policies is mandatory. At the present moment the studies in "Iron Gates" Natural Park on invasive alien species are poor, detailed observation and analyses being needed.

The purpose of this study is an inventory and distribution of invasive alien species, in "Iron Gates" Natural Park, especially to highlight their origins, the most aggressive alien species, and their impact on conservation status of habitats, and indirectly their economic and sociological impact on the human communities. The paper may have an important role in improving the efficiency of conservation measures, offering valuable information to authorities involved in protected areas administration.

\section{MATERIAL AND METHODS}

The observation and analysis of invasive plant species was made in different phoenological phases of the growing season, and by the botanical material collected, preserved and determined from May to September 2012. For a complex analysis of the flora were used chorological data from Romanian Flora, I-XII, bibliographical data extracted from monographic studies of the region and the herbarium sheets (Timişoara, Cluj) collected by various botanists.

Taxa nomenclature is given by Flora Europaea (***, http: //ww2.bgbm.org/europlusmed/), and with Romanian identification field books (Ciocârlan, 2000).

Observation and analyses on fauna were conducted for six years, in various field trips between 2008 and 2014, for Management Plan of "Iron Gates” Natural Park (2013) and also based on scientific literature targeting our study area.

The origin of invasive alien species was established using international databases as http://www.cabi.org/; http://www.invasiveplantatlas.or/; http://www.europe-aliens.org/ and http://www.nobanis.org. We considered it to be essential to represent graphically their native distribution around the world by biogeographical realms and continents of the world.

For a better understanding of the impact on native habitats and species in "Iron Gates" Natural Park a synthetic assessment was necessary. The assessment of the impact of invasive alien species was based on criteria including occurrence, aggressiveness upon native species, the potential to transform and possibly replace the native habitats, the impact on human health and environmental factors. In order to estimate the impact of invasive alien species we used a scale from one to five ( 1 - low occurrence, 2 - low impact, 3 - medium impact, 4 - strong impact and 5 - very high impact) and we managed to construct a list with the most aggressive species in our study area. Further we illustrated their distribution in "Iron Gates” Natural Park to highlight the most vulnerable areas.

\section{RESULTS AND DISCUSSION}

Based on our field observation, data provided by IAS international database and analyses we identified so far 43 invasive alien species (two mammals, seven aquatic invertebrates, four fish and 32 plants) and we synthesized in table 1 their origin, pathways of introduction and their impact in "Iron Gates" Natural Park. 
Table 1: Invasive species of "Iron Gates" Nature Park - origin, pathways of introduction and their impact.

\begin{tabular}{|c|c|c|c|c|}
\hline $\begin{array}{l}\text { Invasive } \\
\text { species }\end{array}$ & $\begin{array}{c}\text { Origin - } \\
\text { Biogeographi-cal } \\
\text { Realm }\end{array}$ & $\begin{array}{l}\text { Pathways of } \\
\text { introduction in } \\
\text { IGNP }\end{array}$ & $\begin{array}{l}\text { Impact } \\
\text { in IGNP }\end{array}$ & $\begin{array}{c}\text { Impact } \\
\text { assessment in } \\
\text { IGNP 1-5 } \\
\text { (low occurrence - } \\
\text { high impact) } \\
\end{array}$ \\
\hline \multicolumn{5}{|l|}{ Mammals } \\
\hline $\begin{array}{l}\text { Myocastor } \\
\text { coypus } \\
\text { Molina, } 1782\end{array}$ & $\begin{array}{l}\text { Neotropic (South } \\
\text { America) }\end{array}$ & $\begin{array}{l}\text { Anthropic - Fur } \\
\text { exploitation }\end{array}$ & $\begin{array}{l}\text { Habitat } \\
\text { degradation, pest }\end{array}$ & 2 \\
\hline $\begin{array}{l}\text { Neovison } \\
\text { vison } \\
\text { Schreber, } 1777\end{array}$ & $\begin{array}{ll}\text { Neartic } & \text { (North } \\
\text { America, Central } \\
\text { America) }\end{array}$ & $\begin{array}{l}\text { Anthropic - Fur } \\
\text { exploitation }\end{array}$ & $\begin{array}{l}\text { Competitive } \\
\text { species, voracious } \\
\text { predator }\end{array}$ & 3 \\
\hline \multicolumn{5}{|c|}{ Aquatic invertebrates } \\
\hline $\begin{array}{l}\text { Corbicula } \\
\text { fluminea } \\
\text { O. F. Müller, } \\
1774\end{array}$ & $\begin{array}{l}\text { Indo-Malay, } \\
\text { Australasia, } \\
\text { Afrotropical } \\
\text { (Asia, Australia, } \\
\text { Africa) }\end{array}$ & $\begin{array}{l}\text { Anthropic - ship } \\
\text { ballast }\end{array}$ & $\begin{array}{l}\text { Competitive } \\
\text { species, } \\
\text { infrastructure } \\
\text { degradation }\end{array}$ & 3 \\
\hline $\begin{array}{l}\text { Dreissena } \\
\text { polymorpha } \\
\text { Pallas, } 1771\end{array}$ & $\begin{array}{l}\text { Paleartic } \\
\text { (Europe) }\end{array}$ & $\begin{array}{l}\text { Anthropic - ship } \\
\text { ballast }\end{array}$ & $\begin{array}{l}\text { Competitive } \\
\text { species, } \\
\text { bioaccumulate } \\
\text { pollutants } \\
\end{array}$ & 2 \\
\hline $\begin{array}{l}\text { Eriocheir } \\
\text { sinensis } \\
\text { Milne-Edwards, } \\
1854\end{array}$ & Paleartic (Asia) & $\begin{array}{l}\text { Anthropic - ship } \\
\text { ballast }\end{array}$ & $\begin{array}{l}\text { Competitive } \\
\text { species, parasite } \\
\text { host, } \\
\text { infrastructure } \\
\text { degradation } \\
\end{array}$ & 4 \\
\hline $\begin{array}{l}\text { Eustrongylides } \\
\text { sp. }\end{array}$ & worldwide & $\begin{array}{l}\text { Natural - birds } \\
\text { and fish parasite }\end{array}$ & Parasite & 3 \\
\hline $\begin{array}{l}\text { Hemimysis } \\
\quad \text { anomala } \\
\text { G. O. Sars, } 1907\end{array}$ & $\begin{array}{l}\text { Paleartic } \\
\text { (Europe) }\end{array}$ & $\begin{array}{l}\text { Anthropic - ship } \\
\text { ballast }\end{array}$ & $\begin{array}{l}\text { Habitat } \\
\text { degradation }\end{array}$ & 2 \\
\hline $\begin{array}{l}\text { Katamysis } \\
\text { warpachowskyi } \\
\text { G. O. Sars, } 1893\end{array}$ & $\begin{array}{l}\text { Paleartic } \\
\text { (Europe) }\end{array}$ & $\begin{array}{l}\text { Anthropic - ship } \\
\text { ballast }\end{array}$ & $\begin{array}{l}\text { Impacts on the } \\
\text { zooplankton }\end{array}$ & 2 \\
\hline $\begin{array}{l}\text { Orconectes } \\
\text { limosus } \\
\text { Rafinesque, } 1817\end{array}$ & $\begin{array}{l}\text { Neartic (North } \\
\text { America) }\end{array}$ & $\begin{array}{l}\text { Anthropic - ship } \\
\text { ballast, } \\
\text { aquaculture }\end{array}$ & $\begin{array}{l}\text { Competitive } \\
\text { species, disease- } \\
\text { crayfish plague }\end{array}$ & 2 \\
\hline
\end{tabular}


Table 1 (continued): Invasive species of “Iron Gates” Nature Park - origin, pathways of introduction and their impact.

\begin{tabular}{|c|c|c|c|c|}
\hline $\begin{array}{c}\text { Invasive } \\
\text { Species }\end{array}$ & $\begin{array}{c}\text { Origin - } \\
\text { Biogeographical } \\
\text { Realm }\end{array}$ & $\begin{array}{l}\text { Pathways of } \\
\text { introduction in } \\
\text { IGNP }\end{array}$ & $\begin{array}{l}\text { Impact } \\
\text { in IGNP }\end{array}$ & $\begin{array}{c}\text { Impact } \\
\text { assessment in } \\
\text { IGNP } \\
1-5 \text { (low } \\
\text { occurrence -high } \\
\text { impact) }\end{array}$ \\
\hline \multicolumn{5}{|l|}{ Fish } \\
\hline $\begin{array}{l}\text { Ameiurus } \\
\text { nebulosus } \\
\text { Lesueur, } 1819 \\
\end{array}$ & $\begin{array}{ll}\text { Neartic } & \text { (North } \\
\text { America) } & \end{array}$ & $\begin{array}{l}\text { Anthropic } \\
\text { aquaculture }\end{array}$ & $\begin{array}{l}\text { Competitive } \\
\text { species }\end{array}$ & 4 \\
\hline $\begin{array}{l}\text { Carassius } \\
\text { auratus gibelio } \\
\text { Bloch, } 1782\end{array}$ & $\begin{array}{l}\text { Paleartic (Europe, } \\
\text { Asia) }\end{array}$ & $\begin{array}{l}\text { Anthropic } \\
\text { aquaculture }\end{array}$ & $\begin{array}{l}\text { Degrading } \\
\text { environmental } \\
\text { conditions and } \\
\text { reproductive } \\
\text { competition } \\
\end{array}$ & 3 \\
\hline $\begin{array}{l}\text { Lepomis gibbosus } \\
\text { Linnaeus, } 1758\end{array}$ & $\begin{array}{ll}\text { Neartic } & \text { (North } \\
\text { America) } & \end{array}$ & $\begin{array}{l}\text { Anthropic } \\
\text { aquarium trade }\end{array}$ & $\begin{array}{l}\text { Competitive } \\
\text { species, reduce } \\
\text { zooplankton }\end{array}$ & 4 \\
\hline \begin{tabular}{ll}
\multicolumn{2}{l}{ Pseudorasbora } \\
parva & Temminck \\
and & Schlegel, \\
1846 & \\
\end{tabular} & Paleartic (Asia) & $\begin{array}{l}\text { Anthropic } \\
\text { aquaculture }\end{array}$ & $\begin{array}{l}\text { Competitive } \\
\text { species, decrease } \\
\text { or extinction of } \\
\text { native cyprinids }\end{array}$ & 2 \\
\hline \multicolumn{5}{|l|}{ Plants } \\
\hline $\begin{array}{l}\text { Acorus calamus } \\
\text { L. }\end{array}$ & $\begin{array}{l}\text { Indo-Malay } \\
\text { (Asia) }\end{array}$ & $\begin{array}{l}\text { Anthropic - plant } \\
\text { trade }\end{array}$ & $\begin{array}{l}\text { Replace native } \\
\text { species }\end{array}$ & 2 \\
\hline $\begin{array}{l}\text { Ailanthus } \\
\text { altissima } \\
\text { (P. Mill) Swingle }\end{array}$ & Paleartic (Asia) & $\begin{array}{ll}\text { Anthropic } & - \\
\text { ornamental } & \\
\text { purposes }\end{array}$ & $\begin{array}{l}\text { Displace native } \\
\text { vegetation, } \\
\text { competitive } \\
\text { species which } \\
\text { reduce } \\
\text { biodiversity }\end{array}$ & 5 \\
\hline $\begin{array}{l}\text { Amaranthus } \\
\text { retroflexus L. }\end{array}$ & $\begin{array}{l}\text { Neartic, } \\
\text { Neotropical } \\
\text { (North America, } \\
\text { Central America) }\end{array}$ & $\begin{array}{l}\text { Anthropic - plant } \\
\text { trade }\end{array}$ & $\begin{array}{l}\text { Aggressive and } \\
\text { competitive weed }\end{array}$ & 2 \\
\hline
\end{tabular}


Table 1 (continued): Invasive species of "Iron Gates” Nature Park - origin, pathways of introduction and their impact.

\begin{tabular}{|c|c|c|c|c|}
\hline $\begin{array}{l}\text { Invasive } \\
\text { Species }\end{array}$ & $\begin{array}{c}\text { Origin - } \\
\text { Biogeographi-cal } \\
\text { Realm }\end{array}$ & $\begin{array}{l}\text { Pathways of } \\
\text { introduction in } \\
\text { IGNP }\end{array}$ & $\begin{array}{l}\text { Impact } \\
\text { in IGNP }\end{array}$ & $\begin{array}{c}\text { Impact } \\
\text { assessment in } \\
\text { IGNP } \\
\text { 1-5 (low } \\
\text { occurrence - high } \\
\text { impact) }\end{array}$ \\
\hline \multicolumn{5}{|l|}{ Plants } \\
\hline $\begin{array}{l}\text { Ambrosia } \\
\text { artemisiifolia L., } \\
1828\end{array}$ & $\begin{array}{ll}\text { Neartic } & \text { (North } \\
\text { America) } & \end{array}$ & $\begin{array}{l}\text { Anthropic } \quad- \\
\text { agriculture }\end{array}$ & $\begin{array}{l}\text { Reduce } \\
\text { biodiversity, } \\
\text { replace native } \\
\text { species, can be } \\
\text { allergenic } \\
\text { species, can host } \\
\text { fungi and viruses } \\
\text { responsible for } \\
\text { crop diseases }\end{array}$ & 3 \\
\hline $\begin{array}{l}\text { Amorpha } \\
\text { fruticosa } \mathrm{L} .\end{array}$ & $\begin{array}{ll}\text { Neartic } & \text { (North } \\
\text { America) } & \end{array}$ & $\begin{array}{lr}\text { Anthropic } & - \\
\text { ornamental } & \\
\text { purposes } & \text { and } \\
\text { significant } & \\
\text { importance } & \text { for } \\
\text { beekeeping } & \\
\end{array}$ & $\begin{array}{l}\text { Competitive } \\
\text { species, it has the } \\
\text { potential to } \\
\text { replace native } \\
\text { vegetation }\end{array}$ & 5 \\
\hline $\begin{array}{l}\text { Asclepias syriaca } \\
\text { Blanco, } 1837\end{array}$ & $\begin{array}{ll}\text { Neartic } & \text { (North } \\
\text { America) } & \end{array}$ & $\begin{array}{l}\text { Anthropic - plant } \\
\text { trade }\end{array}$ & $\begin{array}{lr}\text { Aggressive } & \text { weed, } \\
\text { contains } & \text { toxic } \\
\text { substances } & \text { that } \\
\text { may } & \text { cause } \\
\text { poisoning } & \\
\end{array}$ & 3 \\
\hline $\begin{array}{l}\text { Azolla filiculoides } \\
\text { Lam. }\end{array}$ & $\begin{array}{l}\text { Neartic, } \\
\text { Neotropical } \\
\text { (North America, } \\
\text { Central America, } \\
\text { South America) }\end{array}$ & $\begin{array}{l}\text { Anthropic - plant } \\
\text { trade }\end{array}$ & $\begin{array}{l}\text { Low occurrence } \\
\text { in } \quad \text { Lemnetea } \\
\text { communities }\end{array}$ & 2 \\
\hline $\begin{array}{l}\text { Bidens frondosa } \\
\text { L. }\end{array}$ & $\begin{array}{lr}\text { Neartic } & \text { (North } \\
\text { America, } & \text { Central } \\
\text { America) } & \end{array}$ & $\begin{array}{l}\text { Anthropic - plant } \\
\text { trade }\end{array}$ & $\begin{array}{l}\text { Ecosystem } \\
\text { degradation, } \\
\text { replace native } \\
\text { species }\end{array}$ & 2 \\
\hline $\begin{array}{l}\text { Bidens vulgata } \\
\text { Greene }\end{array}$ & $\begin{array}{ll}\text { Neartic } & \text { (North } \\
\text { America) } & \end{array}$ & $\begin{array}{l}\text { Anthropic - plant } \\
\text { trade }\end{array}$ & $\begin{array}{l}\text { Competitive } \\
\text { species, replace } \\
\text { native species }\end{array}$ & 2 \\
\hline
\end{tabular}


Table 1 (continued): Invasive species of “Iron Gates” Nature Park - origin, pathways of introduction and their impact.

\begin{tabular}{|c|c|c|c|c|}
\hline $\begin{array}{l}\text { Invasive } \\
\text { Species }\end{array}$ & $\begin{array}{c}\text { Origin - } \\
\text { Biogeographi-cal } \\
\text { Realm }\end{array}$ & $\begin{array}{l}\text { Pathways of } \\
\text { introduction in } \\
\text { IGNP }\end{array}$ & $\begin{array}{l}\text { Impact } \\
\text { in IGNP }\end{array}$ & $\begin{array}{c}\text { Impact } \\
\text { assessment in } \\
\text { IGNP } \\
\text { 1-5 (low } \\
\text { occurrence - high } \\
\text { impact) }\end{array}$ \\
\hline \multicolumn{5}{|l|}{ Plants } \\
\hline $\begin{array}{l}\text { Conyza } \\
\text { canadensis (L.) } \\
\text { Cronq. }\end{array}$ & $\begin{array}{ll}\text { Neartic } & \text { (North } \\
\text { America) } & \end{array}$ & $\begin{array}{l}\text { Anthropic - plant } \\
\text { trade }\end{array}$ & $\begin{array}{l}\text { Ecosystem } \\
\text { degradation, } \\
\text { affects crops, can } \\
\text { host for some } \\
\text { plant viruses, } \\
\text { nematodes, fungi } \\
\text { and insects }\end{array}$ & 2 \\
\hline $\begin{array}{l}\text { Cyperus odoratus } \\
\text { L. }\end{array}$ & $\begin{array}{ll}\text { Neartic } & \text { (North } \\
\text { America) } & \end{array}$ & uncertain & $\begin{array}{l}\text { Displace native } \\
\text { vegetation }\end{array}$ & 2 \\
\hline $\begin{array}{l}\text { Dysphania } \\
\text { ambrosioides L. }\end{array}$ & $\begin{array}{l}\text { Neartic, } \\
\text { Neotropical } \\
\text { (Central America, } \\
\text { South America) }\end{array}$ & $\begin{array}{l}\text { Anthropic - plant } \\
\text { trade }\end{array}$ & $\begin{array}{l}\text { Replace native } \\
\text { species }\end{array}$ & 1 \\
\hline $\begin{array}{l}\text { Echinocystis } \\
\text { lobata (Michx.) } \\
\text { Torr. and A. Gray }\end{array}$ & $\begin{array}{ll}\text { Neartic } & \text { (North } \\
\text { America) } & \end{array}$ & $\begin{array}{l}\text { Anthropic - plant } \\
\text { trade decorative } \\
\text { purposes }\end{array}$ & $\begin{array}{l}\text { Ecosystem } \\
\text { degradation, } \\
\text { replace native } \\
\text { species }\end{array}$ & 2 \\
\hline $\begin{array}{lr}\text { Elodea } & \text { nuttallii } \\
\text { (Planch.) } & \text { H. St. } \\
\text { John } & \end{array}$ & $\begin{array}{ll}\text { Neartic } & \text { (North } \\
\text { America) } & \end{array}$ & $\begin{array}{l}\text { Anthropic - plant } \\
\text { trade, aquarium }\end{array}$ & $\begin{array}{l}\text { Reduce } \\
\text { biodiversity, } \\
\text { replace native } \\
\text { species }\end{array}$ & 5 \\
\hline $\begin{array}{l}\text { Elodea } \\
\text { Canadensis Rich. } \\
\text { in Michx. }\end{array}$ & $\begin{array}{ll}\text { Neartic } & \text { (North } \\
\text { America) } & \end{array}$ & $\begin{array}{l}\text { Anthropic - plant } \\
\text { trade, aquarium }\end{array}$ & $\begin{array}{l}\text { Competitive } \\
\text { species, it has the } \\
\text { potential to } \\
\text { replace native } \\
\text { vegetation, } \\
\text { reducing } \\
\text { biodiversity }\end{array}$ & 5 \\
\hline $\begin{array}{l}\text { Erigeron annuus } \\
\text { (L.) Pers }\end{array}$ & $\begin{array}{ll}\text { Neartic } & \text { (North } \\
\text { America) } & \end{array}$ & $\begin{array}{l}\text { Anthropic - plant } \\
\text { trade decorative } \\
\text { purposes }\end{array}$ & $\begin{array}{l}\text { Ecosystem } \\
\text { degradation, } \\
\text { competitive } \\
\text { species }\end{array}$ & 2 \\
\hline $\begin{array}{ll}\text { Erigeron } & \\
\text { strigosus } & \text { Muhl. } \\
\text { ex Willd. } & \\
\end{array}$ & $\begin{array}{ll}\text { Neartic } & \text { (North } \\
\text { America) } & \end{array}$ & $\begin{array}{l}\text { Anthropic - plant } \\
\text { trade }\end{array}$ & Low occurrence & 1 \\
\hline
\end{tabular}


Table 1 (continued): Invasive species of "Iron Gates” Nature Park - origin, pathways of introduction and their impact.

\begin{tabular}{|c|c|c|c|c|}
\hline $\begin{array}{l}\text { Invasive } \\
\text { Species }\end{array}$ & $\begin{array}{c}\text { Origin - } \\
\text { Biogeographi-cal } \\
\text { Realm }\end{array}$ & $\begin{array}{l}\text { Pathways of } \\
\text { introduction in } \\
\text { IGNP }\end{array}$ & $\begin{array}{l}\text { Impact } \\
\text { in IGNP }\end{array}$ & $\begin{array}{c}\text { Impact } \\
\text { assessment in } \\
\text { IGNP } \\
\text { 1-5 (low } \\
\text { occurrence - high } \\
\text { impact) }\end{array}$ \\
\hline \multicolumn{5}{|l|}{ Plants } \\
\hline $\begin{array}{l}\text { Euphorbia } \\
\text { maculata }\end{array}$ & $\begin{array}{ll}\text { Neartic } & \text { (North } \\
\text { America) } & \end{array}$ & uncertain & $\begin{array}{l}\text { Aggressive weed, } \\
\text { ecosystem } \\
\text { degradation }\end{array}$ & 1 \\
\hline $\begin{array}{l}\text { Galinsoga } \\
\text { parviflora } \\
\text { Cav. }\end{array}$ & $\begin{array}{l}\text { Neotropical } \\
\text { (South America) }\end{array}$ & $\begin{array}{l}\text { Antrhopic - plant } \\
\text { trade, agriculture }\end{array}$ & $\begin{array}{l}\text { Habitat } \\
\text { degradation, } \\
\text { reduce crops } \\
\text { productivity }\end{array}$ & 1 \\
\hline $\begin{array}{l}\text { Hibiscus trionum } \\
\text { L. }\end{array}$ & $\begin{array}{l}\text { Paleartic (Asia } \\
\text { Africa) }\end{array}$ & $\begin{array}{l}\text { Anthropic - plant } \\
\text { trade }\end{array}$ & $\begin{array}{l}\text { Intoxication in } \\
\text { livestocks, human } \\
\text { allergies }\end{array}$ & 1 \\
\hline $\begin{array}{l}\text { Juncus tenuis } \\
\text { Willd. }\end{array}$ & $\begin{array}{ll}\text { Neartic } & \text { (North } \\
\text { America) } & \\
\end{array}$ & $\begin{array}{l}\text { Anthropic - plant } \\
\text { trade }\end{array}$ & Low occurrence & 1 \\
\hline $\begin{array}{l}\text { Oenothera } \\
\text { biennis } \\
\text { L. }\end{array}$ & $\begin{array}{ll}\text { Neartic } & \text { (North } \\
\text { America) } & \end{array}$ & $\begin{array}{l}\text { Anthropic } \\
\text { hoticulture, } \\
\text { medical purposes }\end{array}$ & $\begin{array}{l}\text { Habitat } \\
\text { destruction }\end{array}$ & 2 \\
\hline $\begin{array}{l}\text { Oenothera } \\
\text { parviflora L. }\end{array}$ & $\begin{array}{ll}\text { Neartic } & \text { (North } \\
\text { America) } & \end{array}$ & $\begin{array}{l}\text { Anthropic - } \\
\text { hoticulture, } \\
\text { medical purposes }\end{array}$ & $\begin{array}{l}\text { Habitat } \\
\text { destruction }\end{array}$ & 2 \\
\hline $\begin{array}{l}\text { Panicum capilare } \\
\text { L. }\end{array}$ & $\begin{array}{ll}\text { Neartic } & \text { (North } \\
\text { America) } & \end{array}$ & $\begin{array}{l}\text { Anthropic - plant } \\
\text { trade }\end{array}$ & $\begin{array}{l}\text { Aggressive } \text { weed, } \\
\text { replace native } \\
\text { species }\end{array}$ & 2 \\
\hline $\begin{array}{l}\text { Paspalum } \\
\text { paspalodes } \\
\text { (Michx) Scribn }\end{array}$ & $\begin{array}{l}\text { Neartic, } \\
\text { Afrotropical } \\
\text { (North America, } \\
\text { Africa) }\end{array}$ & $\begin{array}{l}\text { Anthropic - plant } \\
\text { trade }\end{array}$ & $\begin{array}{l}\text { Competitive } \\
\text { species, it has the } \\
\text { potential to } \\
\text { replace native } \\
\text { vegetation, } \\
\text { reduce } \\
\text { biodiversity, can } \\
\text { be alternative } \\
\text { host for fungi, } \\
\text { bacteria, viruses } \\
\text { and nematodes }\end{array}$ & 5 \\
\hline $\begin{array}{l}\text { Polygonum } \\
\text { orientale L. }\end{array}$ & Indo-Malay & $\begin{array}{l}\text { Anthropic - plant } \\
\text { trade }\end{array}$ & Low occurrence & 1 \\
\hline $\begin{array}{l}\text { Portulaca } \\
\text { oleracea L. }\end{array}$ & $\begin{array}{l}\text { Uncertain } \\
\text { possibly Neartic }\end{array}$ & $\begin{array}{l}\text { Anthropic - plant } \\
\text { trade }\end{array}$ & $\begin{array}{l}\text { Aggressive weed, } \\
\text { ecosystem } \\
\text { degradation }\end{array}$ & 2 \\
\hline
\end{tabular}


Table 1 (continued): Invasive species of “Iron Gates” Nature Park - origin, pathways of introduction and their impact.

\begin{tabular}{|c|c|c|c|c|}
\hline Rhus typhina L. & $\begin{array}{ll}\text { Neartic } & \text { (North } \\
\text { America) } & \end{array}$ & $\begin{array}{l}\text { Natural } \\
\text { zoochoric } \\
\text { Anthropic - plant } \\
\text { trade, ornamental } \\
\text { purposes }\end{array}$ & $\begin{array}{l}\text { Habitat } \\
\text { destruction }\end{array}$ & 2 \\
\hline $\begin{array}{l}\text { Robinia } \\
\text { pseudoacacia L. }\end{array}$ & $\begin{array}{ll}\text { Neartic } & \text { (North } \\
\text { America) } & \end{array}$ & $\begin{array}{ll}\text { Anthropic } & - \\
\text { horticulture } & \end{array}$ & \begin{tabular}{lr}
\multicolumn{3}{l}{ Competitive } \\
species, it has the \\
potential to \\
replace native \\
vegetation
\end{tabular} & 5 \\
\hline $\begin{array}{l}\text { Sorghum } \\
\text { halepense } \\
\text { (L.) Pers }\end{array}$ & $\begin{array}{l}\text { Paleartic (Europe, } \\
\text { Asia) }\end{array}$ & $\begin{array}{l}\text { Anthropic - plant } \\
\text { trade }\end{array}$ & $\begin{array}{l}\text { Aggressive and } \\
\text { competitive weed } \\
\text { in wetlands } \\
\text { communities, can } \\
\text { cause intoxication } \\
\text { in livestocks, } \\
\text { human allergies }\end{array}$ & 1 \\
\hline $\begin{array}{l}\text { Vallisneria } \\
\text { spiralis } \\
\text { Linnaeus }\end{array}$ & $\begin{array}{l}\text { Paleartic, Indo- } \\
\text { Malay (Europe, } \\
\text { Africa, Asia) }\end{array}$ & $\begin{array}{l}\text { Anthropic - plant } \\
\text { trade, aqurium }\end{array}$ & $\begin{array}{l}\text { Competitive } \\
\text { species, it has the } \\
\text { potential to } \\
\text { replace native } \\
\text { vegetation } \\
\text { Negative impact } \\
\text { on navigation, } \\
\text { recreation and } \\
\text { agriculture } \\
\text { activities }\end{array}$ & 4 \\
\hline $\begin{array}{l}\text { Xanthium } \\
\text { italicum } \\
\text { Moretti }\end{array}$ & $\begin{array}{l}\text { Neotropical } \\
\text { (Central America, } \\
\text { South America) }\end{array}$ & $\begin{array}{l}\text { Anthropic - plant } \\
\text { trade }\end{array}$ & \begin{tabular}{l}
\multicolumn{2}{l}{ Competitive } \\
species, replace \\
nativer species, \\
can rause \\
poisoning and \\
allergies; invades \\
crops
\end{tabular} & 3 \\
\hline
\end{tabular}

The invasive alien species identified in "Iron Gates" Natural Park have origins in six of the biogeographical realms. The biggest proportion $-62.79 \%$ of identified alien species have a Neartic origin, followed by Palearctic biogeographical origin with $20.93 \%$, Neotropical origin with $13.95 \%$, Indo-Malay origin $-9.30 \%$, Afrotropical origin $-4.65 \%$ and only $2.32 \%$ with an Australasian provenance (Fig. 1). We can also notice that only $2.32 \%$ have a worldwide origin and 2.32\% an uncertain origin (Fig. 1). 
The Neartic origin of the species highlights the trade route between North America and Europe, but also the similar ecological characteristics between these areas which permitted a rapid adaptation of alien species. The pathways of introduction are mainly anthropic (Tab. 1) and are favoured by hydrographic characteristic of the study area, the Danube River being an important artery of communication and transport between Orient and Occident. This fact also explains the Paleartic origins of invasive alien species identified in our study area.

The majority of alien species were transported unintentionally from their natural range to "Iron Gates" Natural Park by ship ballast (e.g. aquatic invertebrates) or plant trade with other plant species transported for economic purposes (e.g. weeds).

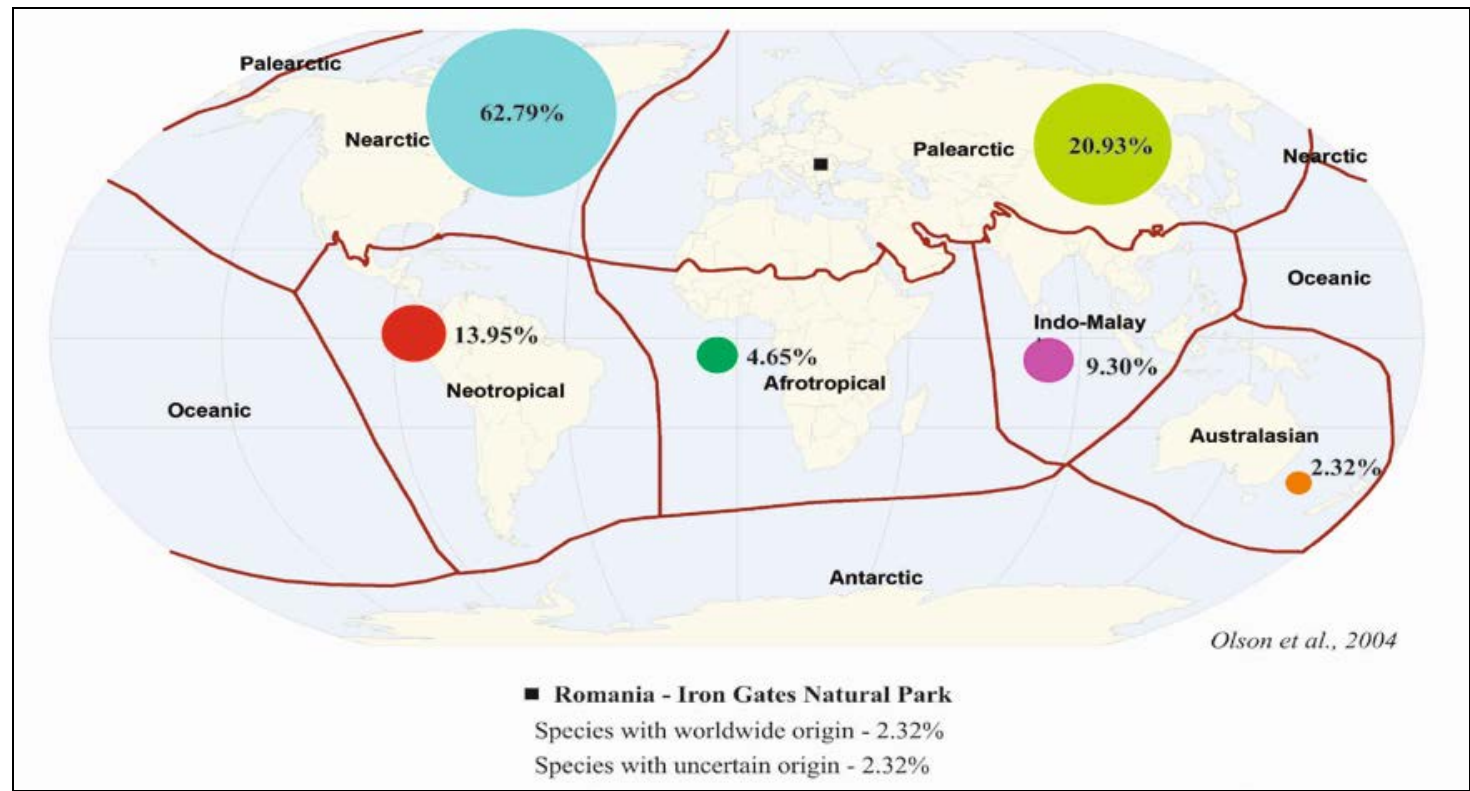

Figure 1: Invasive alien species origins of “Iron Gates” Natural Park by biogeographic realms.

Regarding the origin of invasive species identified in the study area by continents its obviously that the majority has a North American source with $62.79 \%$, followed by Europe and Asia with 16.27\% each, 13.95\% Central America, 11.62\% South America, 9.30\% Africa, 2.32\% Australia (Fig. 2). Only 2.32\% of identified invasive species have a worldwide origin, represented by Eustrongylides sp. - wading birds (Cole, 1999) and freshwater fish parasite (Franson and Custer, 1994). Being a wide spread parasite it makes difficult to establish its accurate origin. We identified one species whose origin is uncertain (Portulaca oleracea), which represents 2.32\% of all identified invasive alien species in "Iron Gates" Natural Park. It is possible for this species to have a Nearctic origin (Chapman et al., 1974).

Some of the identified species were introduced intentionally for use in aquaculture, horticulture and economic interests (e.g. Nevison vision for fur exploitation; Ailanthus altissima for ornamental purposes (Hu, 1979)).

Even thought alien species have been entering Europe for centuries, them have significantly risen in the last 50 years, mainly as a result of increased transportation, trade, travel and other components of globalization (Evangelista and Kumar, 2011; Mack et al., 2000). Studies estimate that the number of IAS in Europe has increased by as much as $76 \%$ since the 1970s (EEA, 2014). The number is likely to continue to grow unless major actions are taken to combat invasion. 


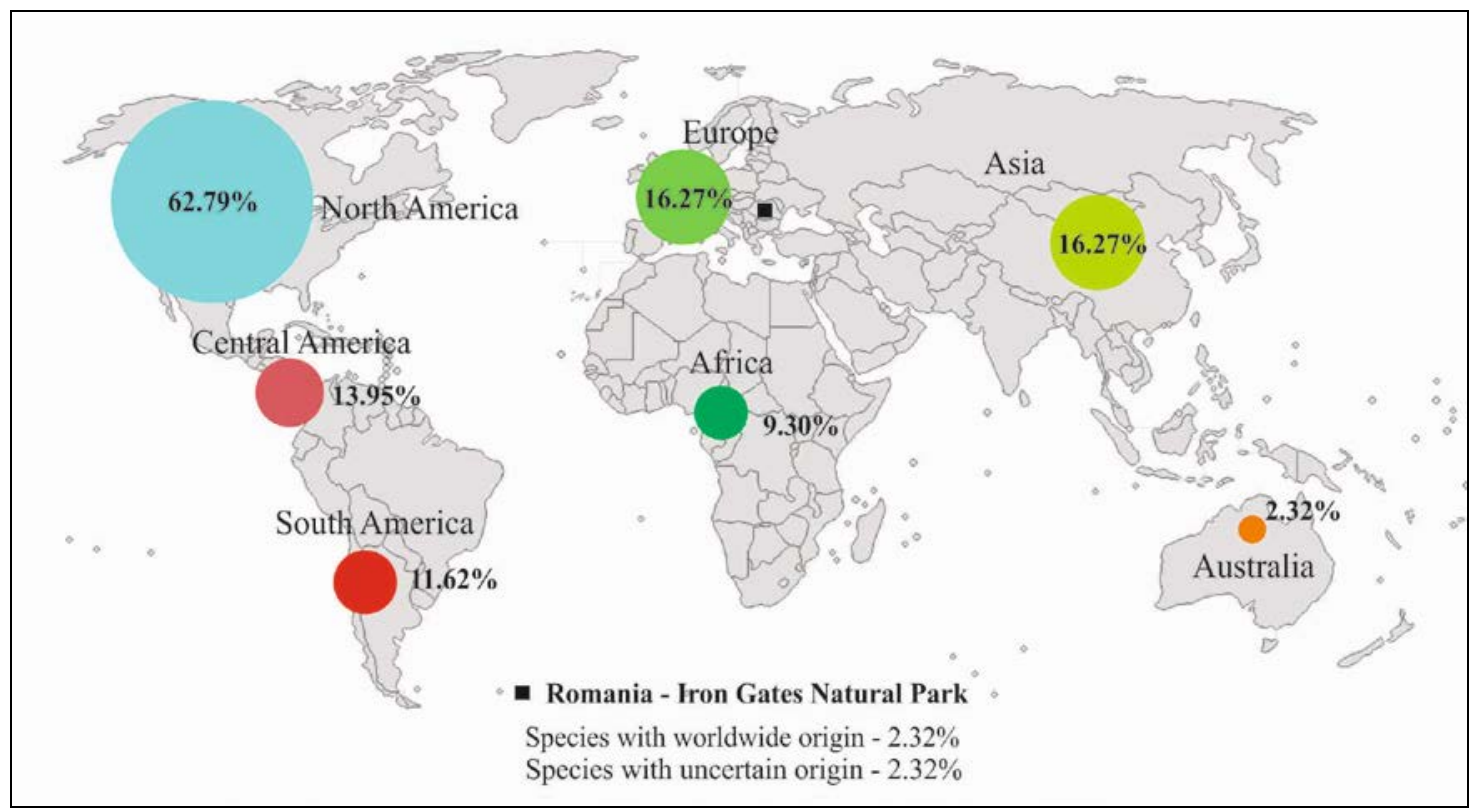

Figure 2: Invasive alien species origins of “Iron Gates” Natural Park by world continents.

In the absence of the data regarding the population of invasive alien species in "Iron Gates” Natural Park on a long period of time, we can only assume that the trend of IAS is similar to Europe.

It was estimated that only $0.1 \%$ introduced plants by humans outside their native area become invasive (Wiliamson, 1996; Williamson and Fitter, 1996). The invasive plants are naturalized plants that are able to spread to a considerable distance from the place of introduction (approximately $>100 \mathrm{~m} / 50$ years for plants that reproduce by seeds; > six m/three years for vegetative reproduction).

Assessing the impact of invasive alien species revealed 10 aggressive species with a high negative impact on the habitats of "Iron Gates" Natural Park: one aquatic invertebrate Eriocheir sinensis, two fish - Ameiurus nebulosus and Lemopis gibbosus, and seven plants Ailanthus altissima, Amorpha fruticosa, Elodea nuttallii, Elodea canadensis, Paspalum paspalodes, Robinia pseudoacacia and Vallisneria spiralis (Fig. 3). Their origins are mainly from Neartic biogeographical realm (70\%), from North America. The other 30\% have a Paleartic origin.

Aggressiveness of these 10 species are threatening the existence of native species from "Iron Gates" Natural Park and also the structure and functions of ecosystems because of their competitiveness regarding food and space (Josefsson and Andersson, 2001) and other adaptive mechanisms. One of this species is Ailanthus altissima that forms dense thickets that displace native vegetation and produce toxins that inhibit the growth of other plants. Also in riparian communities, lower plant species richness and phylodiversity were associated to the presence of A. altissima (Constán-Nava, 2012). 


\section{Impact scale assessment}

1-low occurence, 2-low impact, 3-medium impact, 4-strong impact, 5-very high impact

$\begin{array}{llllllll}0 & 1 & 2 & 3 & 4 & 5\end{array}$

\section{Mammals}

Myocastor coypus Molina, 1782 Neovison vison Schreber, 1777 Aquatic invertebrates

Corbicula fluminea O. F. Müller, 1774 Dreissena polymorpha Pallas, 1771 Eriocheir sinensis Milne-Edwards, 1854 Eustrongylidessp.

Hemimysis anomala G.O.Sars, 1907 Katamysis warpachowskyi G.O.Sars, 1893

Orconectes limosus Rafinesque, 1817

Fish

Ameiurus nebulosus Lesueur, 1819

Carassius auratus gibelio Bloch, 1782

Lepomis gibbosus Linnaeus, 1758 Pseudorasbora parva Temminck \& Schlegel, 1846 Plants Acorus calamus $L$. Ailanthus altissima (P. Mill) Swingle Amaranthus retroflexus $L$. Ambrosia artemisiifolia L., 1828 Amorpha fruticosa $L$. Asclepias syriaca Blanco, 1837 Azolla filiculoides Lam. Bidens frondosa $L$. Bidens vulgata Greene Conyza canadensis (L.) Crong. Cyperusodoratus

Dysphania ambrosioides Echinocystis lobata (Michx.) Torr. \& A.Gray Elodea nuttallii (Planch.) H. St. John Elodea canadensis Rich in Michx.

Erigeron annuus (L.) Pers Erigeron strigosus Muhl.ex Willd. Euphorbiamaculata

Galinsoga parviflora Cav. Hibiscus trionum L. Juncus tenuis Willd. Oenothera biennis $L$.

Oenothera parviflora $L$. Panicum capilare $L$. Paspalum paspalodes (Michx) Scribn

Polygonumorientale $L$. Portulaca oleracea L. Rhustyphina L.

Robinia pseudoacacia L. Sorghum halepense (L.) Pers Vallisneria spiralis Linnaeus,

Xanthium itralicusm Moretti

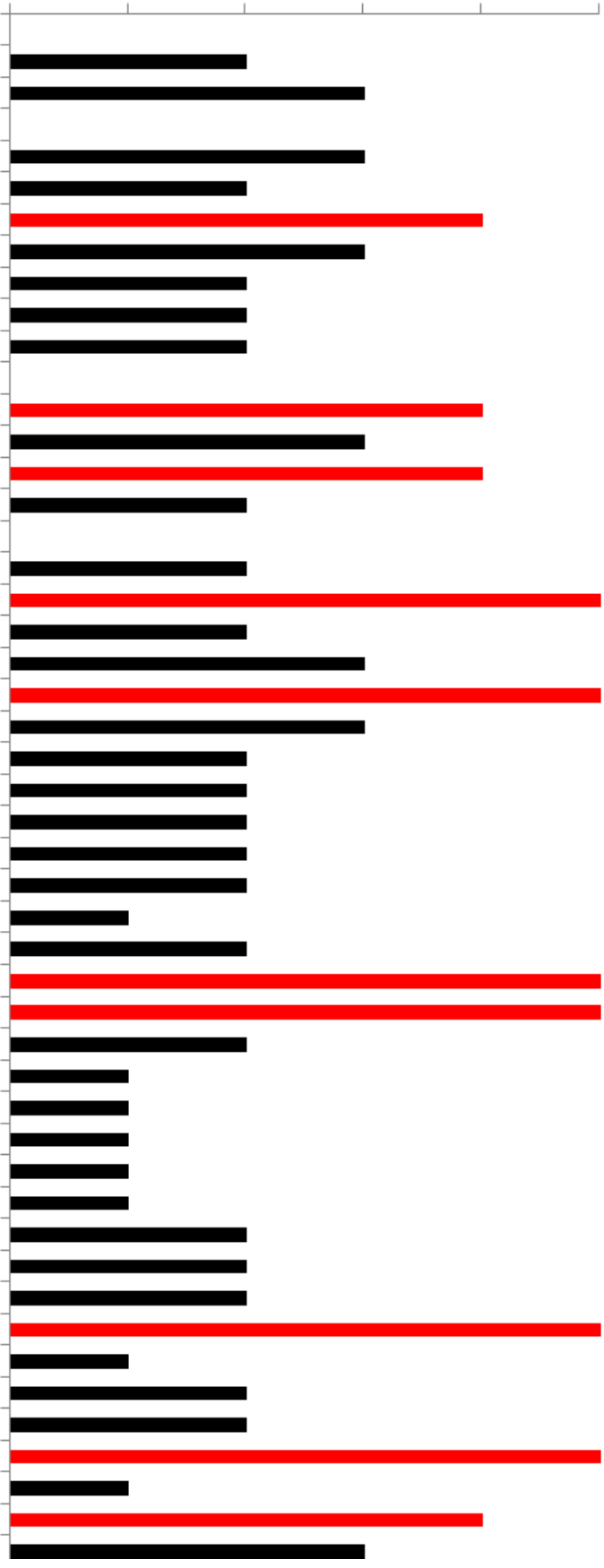

Figure 3: Impact assessment of invasive alien species in IGNP

- highlighting the most aggressive IAS. 
The main issue with invasive alien species, especially with terrestrial (Ailanthus altissima, Amorpha fruticosa, Paspalum paspalodes, Robinia pseudoacacia) and aquatic plants (Elodea nuttallii, Elodea canadensis, Vallisneria spiralis) is that they grow and reproduce rapidly, causing major disturbance to the areas in which they are present.

Due to their great adaptability, alien plant species compete with native species and creates significant transformations. For example, Amorpha fruticosa spreads along Danube watercourse and negatively effects native vegetation (Salix alba, Phragmites australis). It's a major threat for habitats of priority interest and affects protected bird species as Phalacrocorax pygmeus and Aythya nyroca.

The most vulnerable areas (Fig. 4) are the wetlands (Nera-Baziaș, Calinovăț Island, Pojejena, Moldova Veche Island, Liborajdea-Berzasca, Eșelnița Bay, Orșova Bay), usually dominated by cosmopolite species because they offer similar conditions all over the world.

Water is the most important vector for spreading the invasive species, fact also proved in the "Iron Gates" Natural Park. In high elevated areas where heatstroke is a higher risk and the soil is drier, the vegetation communities are cohesive giving no gaps to non-native species or eliminating them in some cases.

Invasive alien species are a significant and growing problem in all EU Member States, including the area of "Iron Gates" Natural Park. Certain aspects of invasive alien species are managed by a variety of existing laws targeting plant health and animal diseases, wildlife trade (CITES) or the use of alien or locally absent species in aquaculture (EEA, 2014), but the most important factor is to prevent their introduction and spread, especially in vulnerable habitats.

By knowing the origins and pathways of introduction of invasive alien species is an important step in having the right knowledge in order to establish measures of limiting their route of access especially by regulating trade (e.g. borders control). It is also important to raise public awareness by organizing extensive campaigns and given the fact that many of invasive alien species have economic importance, popularizing their use might contribute to economic exploitation purposes and in this way perhaps to their eradication (e.g. Oenothera biennis, Oenothera parviflora can be exploited for medical purposes).

In "Iron Gates" Natural Park have been identified so far 43 invasive alien species (two mammals, seven aquatic invertebrates, four fish and 32 plants) that cause significant changes in community structure of aquatic and terrestrial ecosystems and can also change the relations between indigenous and non-native producers and consumers. It is important to mention that only invasive terrestrial plants can change the habitats, because they have the potential to become part of the habitats for other organisms.

It has also been established a list of 10 aggressive species with a high negative impact on the habitats of "Iron Gates" Natural Park based on criteria as occurrence, aggressiveness upon native species, the potential to transform and possibly replace the native habitats, the impact on human health and environmental factors.

The origin of invasive alien species identified in "Iron Gates" Natural Park is predominantly Holartic, 62.79\% have a Neartic origin, followed by a Paleartic provenance with $20.93 \%$, showing the movements of species and the impact of human actions due to globalization process. 


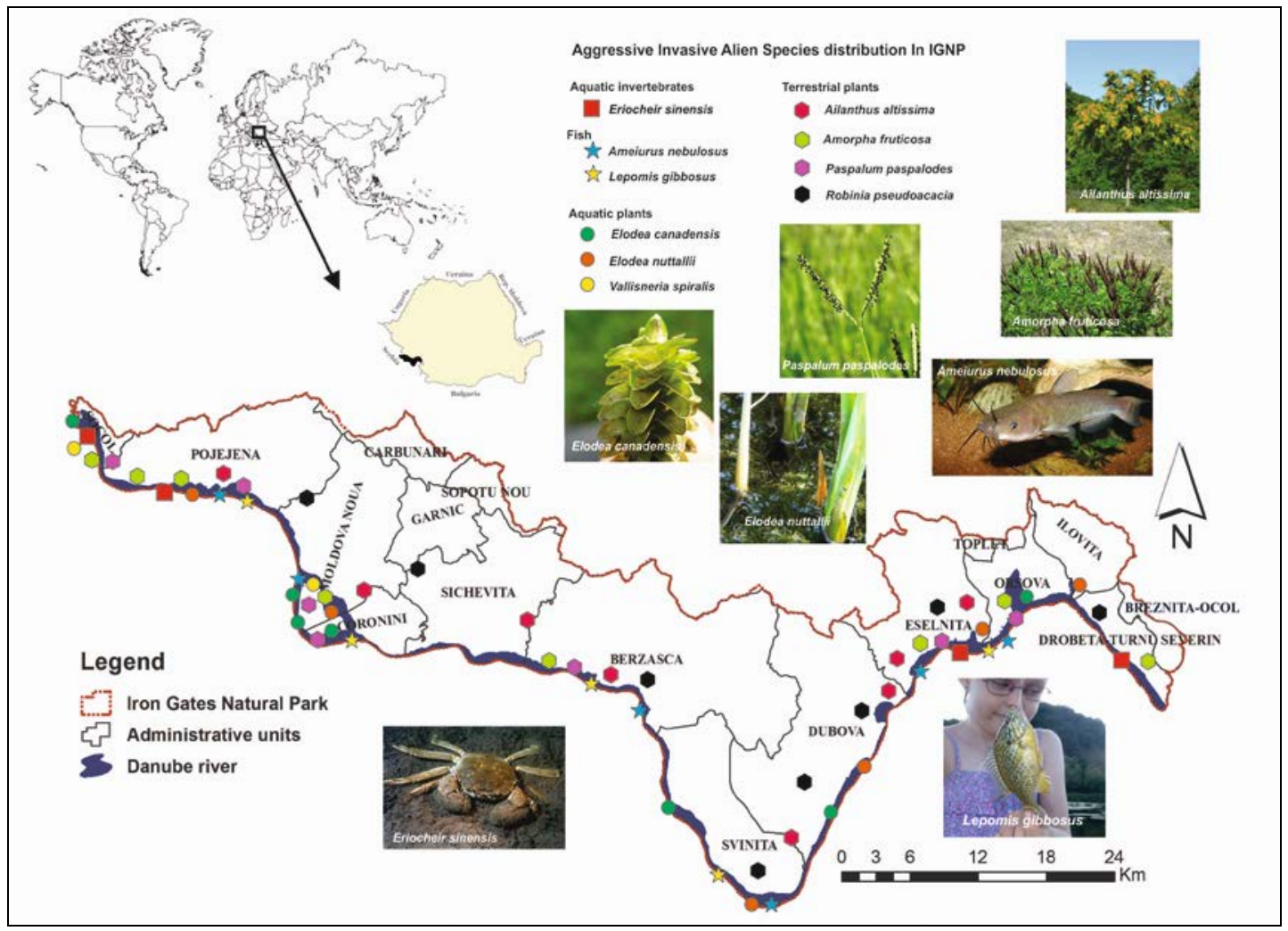

Figure 4: Spatial distribution of most aggressive invasive alien species in "Iron Gates" Natural Park.

A sustainable management of the protected area regarding invasive alien species definitely needs more accurate observations with a longer period of time. This will require more data collection, further detailed observation and analyses that should include observations in all phenological phases of growing season, surveys with professional fishermen, interviews with residents, bird watching and interdisciplinary approach. We highly recommend that the next Management Plan of "Iron Gates" Natural Park needs to establish concrete actions for monitoring invasive alien species, taking into account their diversity, the ecosystems within the park are identified and their aggressiveness. Also, a major objective of the Scientific Council and "Iron Gates" Natural Park Administration should be the emergence of new invasive alien species, and the current state assessment of ecosystems already affected by the their presence.

\section{ACKNOWLEDGEMENTS}

Financial and logistic support for this work was generously provided by the LIFE10 NAT/RO/740 project - Improving the conservation status for the priority species and habitats in the "Iron Gates" wetlands and Centre for Environmental Research and Impact Studies, University of Bucharest. 


\section{REFERENCES}

1. Boiangiu O., 2002 - Studiu climatic al bazinetului Orşova, Drobeta, XI-XII, 220-254, Drobeta Turnu Severin. (in Romanian)

2. Bostan C., Borlea F., Mihoc C. and Beceneaga A. M., 2014 - Spread of Ailanthus altissima species in new areal, Research Journal of Agricultural Science, 46, 1, 104-108.

3. Boşcaiu N., Lupşa V., Resmeriţă I., Coldea G. and Schneider E., 1971 - Vegetaţia lemnoasă mezo-xerotermă (Orno-Cotinetalia) din Defileul Dunării, Ocrotirea naturii, Bucureşti, 15, 1, 49-55. (in Romanian)

4. Călinescu R. and Iana S., 1964 - Consideraţiuni biogeografice asupra Defileului Dunării, Analele Universităţii Bucureşti, Ştiințele naturii, XII, 1, 151-167. (in Romanian)

5. Chapman J., Stewart R. B. and Yarnell R. A., 1974 - Archaeological evidence for preColumbian introduction of Portulaca oleracea and Mullugo verticillata into eastern North America, Economy Botany, 28, 411-412.

6. Ciocârlan V., 2000 - Flora ilustrată a României Pteridophyta et Spermatophyta, Edit. Ceres, Bucureşti, 14-1084. (in Romanian)

7. Cole R. A., 1999 - Chapter 29 - Eustrongylidosis, in Field Manual of Wildlife Diseases, General Field Procedures and Diseases of Birds, Biological Resources Division, 223-228.

8. Constán-Nava S., 2012 - Ecología de la especie invasora Ailanthus altissima (Mill.) Swingle: bases para su control y erradicación en espacios naturales protegidos, PhD Thesis summary, Spain: University of Alicante, http://rua.ua.es/dspace/handle/10045/24861, 4-148. (in Spanish)

9. Daisie (ed.), 2009 - Handbook of Alien Species in Europe, Invading Nature: Springer Series in Invasion Ecology, Springer, Dordrecht, the Netherlands, 133-265.

10. Daleo P., Alberti J. and Iribarne O., 2009 - Biological invasions and the neutral theory, Diversity and Distributions, 15, 547-553.

11. Donaldson J. E., Hui C., Richardson D. M., Robertson M. P., Webber B. L. and Wilson J. R. U., 2014 - Invasion trajectory of alien trees: the role of introduction pathway and planting history, Global Change Biology, 20, 1527-1537.

12. EEA, 2014 - Invasive Alien Species. A European response, EC, Belgium, 5-24.

13. Elton C. S., 1958 - The Ecology of Invasions by Animals and Plants, Methuen, UK, 196.

14. Evangelista P. and Kumar S., 2011 - Trade and transportation is changing the game, Current Zoology, II, 5, 57.

15. Evans J. M., Wilkie A. C. and Burkhardt J., 2008 - Adaptive management of non-native species: moving beyond the either- or through experimental pluralism, Journal of Agricultural and Environmental Ethics, 21, 521-539.

16. Franson J. C. and Custer T. W., 1994 - Prevalence of eustrongylidosis in wading birds from colonies in California, Texas, and Rhode Island, United States of America: Colonial Waterbirds, v, 17, 168-172.

17. Gaertner M., den Breeÿen A., Hui C. and Richardson D. M., 2009 - Impacts of alien plant invasions on species richness: a meta-analysis, Progress in Physical Geography, 33, 319-338.

18. Hu S. Y., 1979 - Ailanthus, Arnoldia, 39, 2, 29-50.

19. Josefsson M. and Andersson B., 2001 - The environmental consequences of alien species in the Swedish lakes Mälaren, Hjälmaren, Vänern and Vättern, Ambio, 30, 8, 514-521.

20. Kumschick S., Bacher S., Dawson W., Heikkilä J., Sendek A., Pluess T., Robinson T. B. and Kühn I., 2012 - A conceptual framework for prioritization of invasive alien species for management according to their impact, NeoBiota, 15, 69-100.

21. Mack R. N., Simberloff D., Lonsdale W. M., Evans H., Clout M. and Bazzaz F. A., 2000 Biotic invasions: causes, epidemiology and control, Ecological Applications, 10, 689-710.

22. Matacă S. Ş., 2005 - Parcul Natural Porţile de Fier, Floră, vegetaţie şi protecţia naturii, Edit. Universitaria, Craiova, 550. (in Romanian) 